\section{Predicting disasters}

\author{
Peter D. Marshall
}

Earthquake Forecasting and Warning.

By Tsuneji Rikitake.

D. Reidel: 1982. Pp.400. Dfl 120, \$45.

EARTHQUAKE prediction, once the domain of astrologers, soothsayers and an elderly lady in Edinburgh, emerged some 15 years ago as an important science with potentially alarming social consequences. Evidence for this is provided by Prof Rikitake in his latest book Earthquake Forecasting and Warning, the third volume in a series Development in Earth and Planetary Sciences which is edited by Rikitake. This new volume is effectively a state of the art report on the prediction programme and in many ways is an update of his earlier book on earthquake prediction

The prediction programme has become a growth industry for geoscientists in several nations, including the USA, USSR, China and for obvious reasons Japan. The international co-operation in this socially important programme is impressive: US scientists have been allowed in to the USSR to monitor premonitory microearthquakes, thought by many to be important precursors to larger and more hazardous earthquakes but they are not yet allowed in to record seismic activities as part of an arms control agreement. China has welcomed and encouraged exchanges of scientists from many countries to study prediction methods and demonstrate their own prediction strategy.

Although there is much exchange of the observational evidence the success of any prediction programme must uItimately depend on the geoscientists ability to understand and exploit any geophysical, geochemical and biological anomalies which may precede a hazardous earthquake. The Chinese would also include an understanding of the macroscopic anomalies: the premonitory anomalies observed by the eye, nose and ear which they have used so successfully to predict earthquakes. There exists at present no definitive model to explain the observed premonitory phenomena. The dilatancy model still remains a popular explanation but only of the precursors associated with changes in crustal stress. Many large earthquakes occur for which there are no phenomena; clearly the science still has a long way to go.

Scientists not involved in the prediction programme may be unaware of the complexity of making a prediction public. Given a set of premonitory observations the geoscientist faces his most difficult task: the assessment of the probability of when and where a hazardous earthquake will occur. The strategy of prediction is equally complex and will surely vary from country to country. A final decision may be too difficult to make; it is a view held by many geoscientists that there still remains an acute shortage of positive data on which to base a prediction and that what little exists is of questionable validity. If this is so it will take a brave scientist to alert his government of an impending hazardous earthquake.

Given an announced warning of an earthquake, how will the public react? The warning could lead to a chaotic exodus from the threatened area. Imagine the consequences of trying to evacuate San Francisco in a hurry! Paradoxically, as the predictions become more and more successful the panic level could increase dramatically. In California it is claimed that 49 deaths could be attributed directly to the emergency evacuation following the successful prediction of a Bay Area earthquake in February 1979. Strangely no nation has a substantial programme to investigate the reaction of the public to earthquake warnings. Surely there is scope here

\section{IMAGE UNAVAILABLE FOR COPYRIGHT REASONS}

Do reliable phenomena precede an earthquake? Chinese officials inspect damage caused by an earthquake in Jiangsu Province, E. China, in 1979. for research by social scientists. At least, Japan and the USA have introduced legislation to control public response to a warning but only time will show how effective this will be.

At present the success rate of earthquake warnings is not high. The most successful short-term (hours-days) prediction is that of China in which the Haicheng earthquake of 1975 was predicted with sufficient accuracy to alert the high risk region. The shock, fortuitously perhaps, was preceded by a plethora of premonitory phenomena including anomalous animal behaviour. Whilst it is recognized that a real scientific basis existed for the prediction, much use was made of amateur observer participation: a feature of the Chinese programme not seriously exploited in the West.

To date most of the research has centered on the acquisition of premonitory data, more effort is needed on the basic research to produce a fundamental understanding of the physical processes of premonitory phenomena. Plate tectonics may explain why and where some earthquakes occur but researchers should not be constrained by a theory in which a fault is stressed by a force applied some thousands of miles away and transmitted horizontally through plates; vertically applied stress may also play an important role in triggering earthquakes. Perhaps more effort should be focused on making a population safe from the direct and indirect effects of hazardous earthquakes.

Anyone concerned, or with an interest in the problems and consequences of earthquake prediction or warning will find Rikitake's book most readable and an excellent reference book with an extensive collection of observed data and references on all aspects of the prediction programme. It brings the reader up to date with recent progress in Japan, China and the USA placing emphasis not only on the collection of data but on the strategy of prediction in the different countries. At times the book which provides a very comprehensive treatment of the subject reads like a catalogue, which in places it is, but at other times it is quite thought-provoking. Unfortunately by refraining from a critical evaluation of the many premonitory phenomena Rikitake does not give us the benefit of his wide experience in earthquake prediction. But will we soon be able to make short-term predictions of hazardous earthquakes? Rikitake and many other researchers are quite optimistic about the prospect, but I wonder?$$
\text { 竞 }
$$

Peter D Marshall is a Principal Scientific Officer in the Seismology Division of the UK Ministry of Defence, Alder. maston. 